\title{
Analyzing Rigidity with Pebble Games
}

\author{
Audrey Lee ${ }^{1}$ Ileana Streinu ${ }^{2}$ Louis Theran ${ }^{1}$
}

\begin{abstract}
How many pair-wise distances must be prescribed between an unknown set of points, and how should they be distributed, to determine only a discrete set of possible solutions? These questions, and related generalizations, are central in a variety of applications. Combinatorial rigidity shows that in two-dimensions one can get the answer, generically, via an efficiently testable sparse graph property.

We present a video and a web site illustrating algorithmic results for a variety of rigidity-related problems, as well as abstract generalizations. Our accompanying interactive software is based on a comprehensive implementation of the pebble game paradigm.
\end{abstract}

\section{Rigidity Models}

We start with a brief overview of the models of rigidity relevant for the presented work. For each model, we describe the geometric constraints that define it and the rigidity property we want to determine.

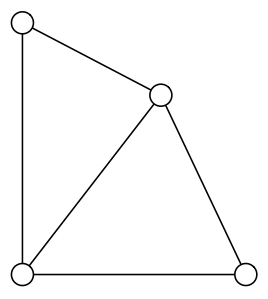

(a)

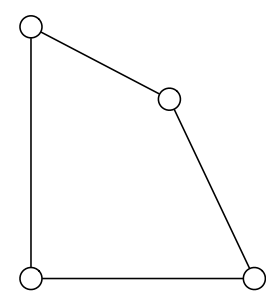

(b)
Figure 1: Bar-and-joint structures in two-dimensions: (a) minimally rigid and (b) flexible.

Planar bar-and-joint rigidity. A bar-and-joint framework consists of fixed-length bars connected by universal joints allowing full rotation of the incident bars. If the only motions maintaining the lengths of all the bars are trivial rigid motions (translations and rotations), the framework is rigid (see Figure 1(a)); oth-

\footnotetext{
${ }^{1}$ Computer Science Department, University of Massachusetts Amherst, USA, \{alee, theran\}@cs.umass.edu

${ }^{2}$ Department of Computer Science, Smith College, Northampton, MA 01063, USA, istreinu@smith.edu.
}

erwise, it is flexible (see Figure 1(b) for an example in two-dimensions).

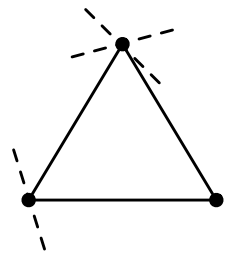

Figure 2: Minimally pinned bar-slider framework.

Planar slider pinning. A bar-and-joint framework in the plane may be additionally constrained by sliders, which force joints to move on fixed lines; we call such structures bar-slider frameworks. Allowed motions preserve the lengths of all the bars and move vertices constrained by sliders along the specified lines. In this model, we are concerned with pinning rigidity: a bar-slider framework is pinned if it is completely immobilized, i.e. rigidly attached to the plane. See Figure 2.

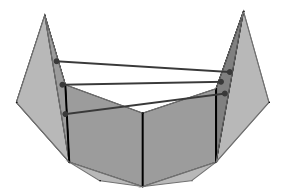

Figure 3: Minimally rigid body-hinge-and-bar structure.

Body-bar and body-hinge rigidity. A body-bar framework consists of rigid bodies connected by fixed length bars; the bars are rigidly attached to the bodies with universal joints. In addition to bars, hinges may also constrain the structure (see Figure 3 for a 3D body-hinge-and-bar structure). They constrain the incident bodies to a relative rotation about the hinge axis. A body-and-bar framework is rigid if the only motions of the framework are trivial ones, as in the bar-and-joint case; otherwise, the framework is flexible.

Parallel redrawings. Given a graph embedded in the plane, a parallel redrawing maintains the direction of each edge. If every parallel redrawing of the graph is 
similar to the original, the graph is said to be direction rigid; otherwise, it is flexible.

\section{Rigidity analysis problems}

For each model of rigidity, we are interested in the following problems; with respect to terminology, framework, rigid and flexible are to be taken in the context of a given model.

Decision: Is a framework rigid?

Extraction: Find a maximum-cardinality set of independent constraints in a framework. For a rigid input, this is a minimally rigid substructure (one which becomes flexible after removing any constraint).

Components: Detect the maximal rigid substructures (called components) of a flexible framework.

Optimization: Extract a set of independent constraints optimizing a given linear weight function.

Extension: Given a flexible framework, describe a set of constraints whose addition would rigidify it. (For example, give a set of sliders that pin a bar-and-joint framework.)

Generic rigidity theorems. The generic rigidity of these models is captured by combinatorial properties. Laman's theorem [2] characterizes minimally rigid 2D bar-and-joint frameworks, and Tay's theorem [8] characterizes minimally rigid body-and-bar frameworks in arbitrary dimension $d$ ([9] and [10] observe that hinges may be represented by 5 bars). For results relating to parallel redrawings, see [10]. In [7], we present results for bar-slider pinning rigidity.

\section{Pebble games and sparsity}

The combinatorial rigidity characterizations are based on hereditary counts on the number of edges in subgraphs. These counts have been generalized to sparse graphs and hypergraphs $[3,5,6,10]$. A (hyper)graph $G$ is $(k, \ell)$-sparse if any set of $n^{\prime}$ vertices spans at most $k n^{\prime}-\ell$ edges in $G$ for non-negative integer parameters $k$ and $\ell$ which allow for non-triavial graphs (e.g. for graphs, they must satisfy $\ell \in[0,2 k)$ ). This definition generalizes to other situations, including graded sparsity [5], in which the edges of $G$ are partitioned into classes, each of which satisfies its own sparsity counts.

Our pebble games, which extend the elegant algorithm of Jacobs and Hendrickson for 2D bar-and-joint rigidity [1], are a family of graph construction rules indexed by non-negative integers $k$ and $\ell$. The $(k, \ell)$-pebble game "recognize" exactly the $(k, \ell)$-sparse graphs [3], and variations on them address all the algorithmic questions listed above. In particular, $(2,3)$-pebble games solve planar rigidity and parallel redrawing questions, $k=\ell=\left(\begin{array}{c}d+1 \\ 2\end{array}\right)$ handle body-bar-hinge structures in dimension $d$, and a combination of $(2,3)$ and $(2,0)$-pebble games solve pinning rigidity in the plane.

The basic $(k, \ell)$-pebble game is played by a single player on a directed graph. It is described in terms of an initial configuration (an empty graph on $n$ vertices, with $k$ pebbles on each) and two allowed moves:

Add edge move: If vertices $i$ and $j$ have at least $\ell+1$ pebbles altogether on them, add the directed edge $i j$ and pick up a pebble from one of the endpoints.

Pebble slide move: If $i j$ is an edge and there is a pebble on $j$, reverse the edge $i j$ and move the pebble from $j$ to $i$.

We have developed several types (including component, colored and graded variations) of pebble games $[3,4,5,6]$ for all sparse graphs and hypergraphs.

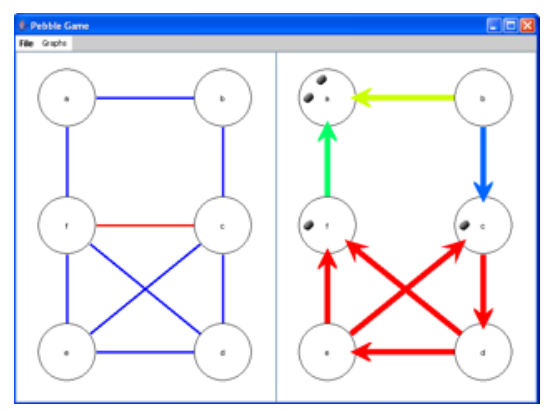

Figure 4: A snapshot of the component pebble game for 2D bar-and-joint rigidity.

Demo site. Applets, demos and an accompanying video can be found at http://linkage.cs . umass.edu/pg. The video may be independently downloaded from http://linkage.cs.umass.edu/pg/ socgSubmission.html.

\section{References}

[1] D. J. Jacobs and B. Hendrickson. An algorithm for two-dimensional rigidity percolation: the pebble game. Jour. Comput. Physics, 137:346-365, November 1997.

[2] G. Laman. On graphs and rigidity of plane skeletal structures. Journal of Engineering Mathematics, 4:331-340, 1970.

[3] A. Lee and I. Streinu. Pebble game algorithms and sparse graphs. Discrete Mathematics, 308(8):1425-1437, 2008.

[4] A. Lee, I. Streinu, and L. Theran. Finding and maintaining rigid components. In Proc. Canadian Conf. Comput. Geometry, 2005.

[5] A. Lee, I. Streinu, and L. Theran. Graded sparse graphs and matroids. Jour. Univ. Comp. Science, 13(10), 2007.

[6] I. Streinu and L. Theran. Sparse hypergraphs and pebble game algorithms. European Journal of Combinatorics, To appear.

[7] I. Streinu and L. Theran. Combinatorial genericity and minimal rigidity. In Proc. 24th Symp. Comput. Geometry (SoCG), 2008.

[8] T.-S. Tay. Rigidity of multigraphs I: linking rigid bodies in n-space. Journal of Combinatorial Theory Series, B 26:95-112, 1984.

[9] T.-S. Tay. Linking ( $\mathrm{n}-2$ )-dimensional panels in n-space II: (n - 2, 2)-frameworks and body and hinge structures. Graphs and Combinatorics, 5:245-273, 1989.

[10] W. Whiteley. Some matroids from discrete applied geometry. In J. Bonin, J. G. Oxley, and B. Servatius, editors, Matroid The- 
ory, volume 197 of Contemporary Mathematics, pages 171-311.

American Mathematical Society, 1996. 